\title{
Birefringence Measurements in Fibers Without Polarizer
}

\author{
LUC THÉVENAZ, JEAN-PAUl PELLAUX, NICOLAS GISIN, AND JEAN-PIERRE VON DER WEID
}

\begin{abstract}
A novel method is described which measures the delay between polarization eigenmodes in either high- or low-birefringence fibers. The main advantage of this interferometric method is its ability to perform the measurements without the use of any polarizing device. This results in a particularly easy handling and makes this technique very suitable for systematic fiber controls. The obtained accuracy using an all-fiber setup is $\mathbf{0 . 0 2} \mathrm{ps} / \mathrm{m}$ for highly birefringent fibers and $\mathbf{0 . 0 0 3}$ $\mathrm{ps} / \mathrm{m}$ when the birefringence is low
\end{abstract}

\section{INTRODUCTION}

$\mathrm{C}$ OHERENT transmission systems offer important potential advantages compared to the classical optical links and are forecast to take a predominant place in the near future. These systems require the light polarization state to be pure and stationary at the fiber output end. This can be achieved by using either very high- or very lowbirefringence fibers [1]. In both cases the knowledge of the birefringence value is important. Before installing future links, the fiber check procedure will therefore include the measurement of birefringence, together with other parameters such as the attenuation and the chromatic dispersion.

The need for a reliable and easy-to-use measurement method for systematic birefringence control has recently given rise to many experimental developments. Numerous methods have been proposed to date measuring either the birefringence or the polarization mode dispersion [2][6]. But all these methods require at least one light polarizing device and most of them retardation plates. Thus, in addition to the fiber positioning, the polarization state must be set in a particular orientation with respect to the fiber birefringence eigenaxes. This supplementary alignment can make the handling uneasy and may result in wrong measurements if inaccurately performed.

A novel method is reported here which performs this measurement without the use of any light polarizing device or retardation plate. This technique uses the funda-

Manuscript received September 24, 1988; revised April 28, 1989. This work was supported by the Swiss PTT and the Swiss National Fund for Scientific Research.

L. Thévenaz was with the Group of Applied Physics, University of Ge neva, 1211 Geneva 4. Switzerland. He is now with EPFL Metrology Lab. Swiss Federal Institute of Technology, 1015 Lausanne, Switzerland.

J.-P. Pellaux and N. Gisin are with the Group of Applied Physics, University of Geneva, 1211 Geneva 4, Switzerland.

J.-P. von der Weid was with the Group of Applied Physics, University of Geneva, 1211 Geneva 4, Switzerland, on leave from the Physics Department, Pontificia Universidade Catholica, Rio de Janeiro 22452, Brazil.

IEEE Log Number 8929045 mental property that an interferometric reconstruction cannot occur for lightwaves in orthogonal polarization states. The interferences can therefore provide a polarization-dependent information even using an unpolarized light source. This was achieved using an interferometer with a reference arm of known birefringence, the other arm being a sample of the fiber to be measured. The birefringence is obtained from either the positions of the interference patterns or their contrast, provided that the light source is polychromatic and wavelength tunable.

\section{BASIC PRINCIPLES}

Assuming a single transverse mode, let the electrical field at the interferometer input be:

$$
E=\sum_{i} E_{o j} e^{j \omega_{i} t} u_{i}
$$

where the summation is performed over the longitudinal modes-or frequencies-of the source and $E_{o i}$ is the mode complex amplitude, $\omega_{i}$ its optical angular frequency, and $u_{i}$ a Jones' vector representing its polarization state.

After the interferometric reconstruction the detected intensity is given by

$$
\begin{aligned}
I= & \frac{1}{2} \epsilon_{0} c\left\{\left|b_{1}\right|^{2} \sum_{i}\left|E_{o i}\right|^{2}+\left|b_{2}\right|^{2} \sum_{i}\left|E_{o i}\right|^{2}\right. \\
& +b_{1} b_{2}^{*} \sum_{i}\left|E_{o i}\right|^{2} e^{j\left(\beta_{i 1} l_{1}-\beta_{i 2} l_{2}\right)} u_{i 2}^{+}\left(l_{2}\right) u_{i 1}\left(l_{1}\right) \\
& \left.+b_{1}^{*} b_{2} \sum_{i}\left|E_{o i}\right|^{2} e^{-j\left(\beta_{i 1} l_{1}-\beta_{i 2} l_{2}\right)} u_{i 1}^{+}\left(l_{1}\right) u_{i 2}\left(l_{2}\right)\right\}
\end{aligned}
$$

where $b_{1}, b_{2}$ are complex coefficients with $\left|b_{1}\right|^{2}+\left|b_{2}\right|^{2}$ $=1$, representing the constant amplitude and phase differences between the fields in each arm, and $\beta_{i 1}, \beta_{i 2}$ are the propagation constants of each mode in each arm. The two last terms represent the interference signal, are complex conjugated and are the only polarization-dependent terms, as anticipated.

The polarization vector $u_{i k}\left(l_{k}\right), k=1,2$, can be related to the incident polarization vector $u_{i}$ by an unitary transformation

$$
u_{i k}\left(l_{k}\right)=\rho_{i k} u_{i}
$$

where $\rho_{i k}$ is an unitary matrix representating the transformation of any polarization state at the frequency $\omega_{i}$ after the propagation through the arm $k$. It therefore takes the 
following general form

$$
\mathcal{P}_{i k}=\mathcal{R}_{i k} \mathcal{B}_{i k} \mathcal{R}_{i k}^{-1} \mathfrak{Q}_{i k}, \quad k=1,2
$$

where

$$
\beta_{i k}=\left(\begin{array}{ll}
e^{-j \phi_{i k}} & 0 \\
0 & e^{j \phi_{i k}}
\end{array}\right), \quad k=1,2
$$

and $\boldsymbol{R}_{i k}, \mathbb{Q}_{i k}$ are rotation matrices.

Expression (4) simply shows that any nonpolarizing transformation of the polarization state can be reduced to the application of an optically active element $\mathfrak{C}_{i k}$ followed by a phase retardation element $\beta_{i k}$ with an orientation defined by $\boldsymbol{R}_{i k}$.

The product $u_{i 2}^{+}\left(l_{2}\right) u_{i 1}\left(l_{1}\right)$ in (2) can now be reexpressed using (3) as

$$
u_{i 2}^{+}\left(l_{2}\right) u_{i 1}\left(l_{1}\right)=u_{i}^{+} \rho_{i 2}^{+} \rho_{i 1} u_{i}
$$

which is, in fact, the average value of the operator $\odot_{i 2}^{+} \mathcal{P}_{i 1}$ in the state $u_{i}$. For unpolarized light the polarization state is not pure, but is a statistical mixture of states. In this case the calculation of an average value is more simply performed using a density operator $\rho$ that contains the probabilities associated with the different polarization states. The average value in (5) is then given by

$$
\left\langle\Theta_{i 2}^{+} \mathcal{Q}_{i 1}\right\rangle=\operatorname{Tr}\left(\rho \boldsymbol{P}_{i 2}^{+} \boldsymbol{P}_{i 1}\right) \text {. }
$$

For completely unpolarized light the density operator $\rho$ is simply

$$
\rho=\left(\begin{array}{cc}
\frac{1}{2} & 0 \\
0 & \frac{1}{2}
\end{array}\right)
$$

Now let

$$
\begin{aligned}
\mathbb{Q}_{i k} & =\left(\begin{array}{cc}
\cos \alpha_{i k} & -\sin \alpha_{i k} \\
\sin \alpha_{i k} & \cos \alpha_{i k}
\end{array}\right) \\
\mathbb{R}_{i k} & =\left(\begin{array}{cc}
\cos \vartheta_{i k} & -\sin \vartheta_{i k} \\
\sin \vartheta_{i k} & \cos \vartheta_{i k}
\end{array}\right)
\end{aligned}
$$

so that expression (6) can be straightforwardly evaluated using (4) and (7), yielding

$$
\begin{aligned}
\left\langle\Theta_{i 2}^{+} \vartheta_{i 1}\right\rangle= & \cos \left(\vartheta_{i 2}-\vartheta_{i 1}\right) \cos \left(\vartheta_{i 2}-\vartheta_{i 1}\right. \\
& \left.-\alpha_{i 2}+\alpha_{i 1}\right) \cos \left(\phi_{i 1}-\phi_{i 2}\right) \\
& +\sin \left(\vartheta_{i 2} \vartheta_{i 1}\right) \sin \left(\vartheta_{i 2}-\vartheta_{i 1}-\alpha_{i 2}+\alpha_{i 1}\right) \\
& \cdot \cos \left(\phi_{i 1}+\phi_{i 2}\right) .
\end{aligned}
$$

This result being a real quantity it represents the product $u_{i 2}^{+}\left(l_{2}\right) u_{i 1}\left(l_{1}\right)$ and its complex conjugate $u_{i 1}^{+}\left(l_{1}\right) u_{i 2}\left(l_{2}\right)$ as well, provided that the incident light is unpolarized.

If one of the arms, say arm 1, is neither birefringent $\left(\phi_{i 1}=0\right)$ nor optically active $\left(\alpha_{i 1}=0\right)$, expression (9) greatly simplifies and reads

$$
\left\langle\Theta_{i 2}^{+} \Theta_{i 1}\right\rangle=\cos \alpha_{i 2} \cos \phi_{i 2} \text {. }
$$

Linear and circular birefringences have therefore the same overall effect on the interferometric reconstruction, be- cause an unpolarized light equally and randomly excites any couple of orthogonal polarization states.

Let us approximate by a Taylor's series expansion the propagation constant $\beta_{i k}$ in each arm

$$
\begin{aligned}
\beta_{i k}= & \beta_{0 k}+\beta_{k}^{\prime} \cdot\left(\omega_{i}-\omega_{0}\right) \\
& +\frac{1}{2} \beta_{k}^{\prime \prime} \cdot\left(\omega_{i}-\omega_{0}\right)^{2}+\cdots k=1,2
\end{aligned}
$$

and the phase retardation $\phi_{i 2}$

$$
\phi_{i 2}=\phi_{0}+\frac{\Delta n}{2 c} \omega_{0} l_{2}+\frac{\Delta N}{2 c}\left(\omega_{i}-\omega_{0}\right) l_{2}+\cdots
$$

where $\phi_{0}$ is a possible frequency-independent phase retardation, $\Delta n$ is the birefringence and $\Delta N=\Delta n-$ $\lambda d \Delta n / d \lambda$ is the equivalent refractive index difference for the group velocity. In a first stage the arm 2 is assumed to be purely birefringent $\left(\alpha_{i 2}=0\right)$, the effect of an optical activity being later deduced by a simple analogy.

The detected intensity given by (2) can be finally calculated using (10), (11), and (12), the summation over the longitudinal modes being converted into an integral by introducing a normalized spectral density

$$
E_{o i}=E_{o} F\left(\omega_{i}-\omega_{0}\right) d \omega_{i}
$$

where $\omega_{0}$ is the spectrum median frequency. Hence

$$
\begin{aligned}
I= & \frac{1}{2} \epsilon_{0} c\left|E_{0}\right|^{2}\left\{1+\left|b_{1}\right|\left|b_{2}\right|\right. \\
& \cdot\left[\left|g_{D}\left(\beta_{1}^{\prime} l_{1}-\left(\beta_{2}^{\prime}+\frac{\Delta N}{2 c}\right) l_{2}\right)\right|\right. \\
& \cdot \cos \left(\beta_{01} l_{1}-\left(\beta_{02}+\omega_{0} \frac{\Delta n}{2 c}\right) l_{2}-\phi_{0}+\phi\right) \\
& +\left|g_{D}\left(\beta_{1}^{\prime} l_{1}-\left(\beta_{2}^{\prime}-\frac{\Delta N}{2 c}\right) l_{2}\right)\right| \\
& \left.\left.\cdot \cos \left(\beta_{01} l_{1}-\left(\beta_{02}-\omega_{0} \frac{\Delta n}{2 c}\right) l_{2}+\phi_{0}+\phi\right)\right]\right\}
\end{aligned}
$$

where $\phi$ is a constant phase including the arguments of $b_{1}, b_{2}^{*}$, and $g_{D}$, and

$$
\begin{aligned}
g_{D}\left(\beta_{1}^{\prime} l_{1}-\beta_{2}^{\prime} l_{2}\right)= & \int\left|F\left(\omega-\omega_{0}\right)\right|^{2} \exp \left[j \frac { 1 } { 2 } \left(\beta_{1}^{\prime \prime} l_{1}\right.\right. \\
& \left.\left.-\beta_{2}^{\prime \prime} l_{2}\right)\left(\omega-\omega_{0}\right)^{2}\right] \\
& \cdot \exp \left[j\left(\beta_{1}^{\prime} l_{1}-\beta_{2}^{\prime} l_{2}\right)\left(\omega-\omega_{0}\right)\right] \\
& \cdot d\left(\omega-\omega_{0}\right)
\end{aligned}
$$

is the first-order degree of temporal coherence of the light at the output of the interferometer, that is, taking into account the smoothing effect of the dispersive terms. Actually this effect is negligible in most cases, the dispersion remaining low over the spectral region of interest. In extreme conditions (large chromatic dispersion and strongly assymmetrical spectrum) $\phi$ is no longer constant and de- 
pends on the chromatic dispersion value and the spectral width.

Expression (14) clearly shows that each polarization mode gives rise to an independent interferometric reconstruction. When the length $l_{1}$ is varied at a constant rate, two contributions to the interference signal are distinguishable for each polarization mode: a rapidly varying contribution, given by the cosine term and representing the individual interferences, and a contrast term given by $\left|g_{D}\right|$ which slowly varies, provided that the source spectral width $\Delta \omega$ is much smaller than $\omega_{0}$. Both these contributions contain information about the birefringence.

The maximum of contrast occurs when the group delays in both arms are equal. This property is widely used to measure the chromatic dispersion [7]. As intuitively expected, the effect of birefringence is to split the interference pattern obtained when the arm length $l_{1}$ is varied into two new patterns. Similarly, if arm 2 is in addition optically active, each of these patterns is split again, resulting in four independent patterns.

\section{Experimental Setup}

The experimental setup, previously developed for chromatic dispersion measurements [7], can be used unmodified for birefringence measurements. For clarity a diagram of the setup is shown in Fig. 1 and its main features are briefly described as follows.

- A Michelson configuration, in which one single-mode coupler performs the light splitting and the reconstruction, resulting in fewer optical elements, a double optical path through the test fiber, and thus better stability and resolution.

- A fiber variable delay line in the reference arm, achieved using the elastic properties of the fiber and enabling the length $l_{1}$ of the reference arm to be scanned without using an air path.

These features enable an all-fiber setup to be achieved, resulting in a better reliability and a great immunity to external perturbations.

The light source is a tungsten halogen lamp and a part of its spectrum is selected using a monochromator. While measuring, the reference arm length $l_{1}$ is varied at a constant speed, so that the light experiences a Doppler shift. The interferometric reconstruction therefore gives rise to a beat signal, of which the amplitude can be measured using a tuned lock-in amplifier. This amplitude is a direct and fast measurement of the interference contrast.

The obtained 2-fs time accuracy on the maximum contrast position corresponds to a $0.5 \mathrm{ps} / \mathrm{km}$ group delay resolution using a $1.85-\mathrm{m}$ test fiber. The all-fiber configuration requires two calibrations: one to establish the relationship between the actual elongation and the optical path change in the fiber delay line and the other to determine the dispersion spectrum of the reference arm. This latter calibration is achieved by replacing the test fiber by a nondispersive air path. A non-negligible birefringence in the reference arm can also be detected by this calibration,

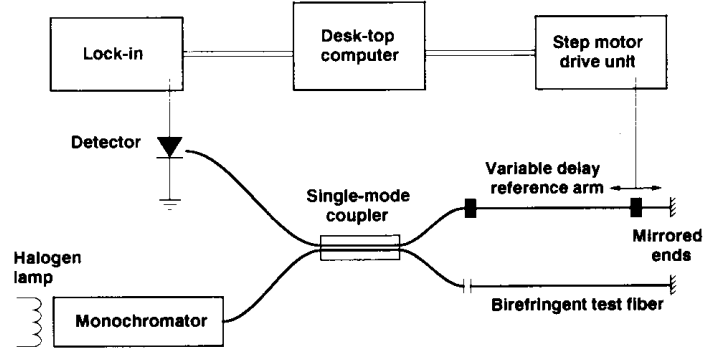

Fig. 1. Schematic diagram of the experimental setup.

so that a careful selection of suitable reference fiber can be performed (more complete information can be found in [7]).

\section{Results}

\section{A. High-Birefringence Fibers}

When the delay between the polarization modes is large enough to exceed the coherence length, the interference patterns are well distinct so that independent group delay measurements can be performed on each pattern. The interferences' visibility in such a situation is shown in Fig. 2 , when the length of the reference arm is varied. The maximum visibilities at the center of each pattern must be equal, provided that the light is perfectly unpolarized. Actually the partial polarizing effect of the monochromator gives rise to a small difference of visibility, as illustrated in Fig. 2.

The group delay spectrum can therefore be measured independently for each polarization mode, as shown in Fig. 3, and their chromatic dispersion can be separately calculated. The difference between the two group delay curves yields the polarization mode dispersion $\Delta N / c$, shown in Fig. 4 for two fibers with a different design to induce the birefringence. An increased scattering of the experimental points is observed for the longer wavelengths, owing to the greater overlap of the interference patterns due to a larger coherence length. The overlapping of the interference patterns strongly perturbates the measurement and actually limits the minimum polarization mode dispersion which can be accurately measured. The lower bound can be defined similarly to the resolving power of an optical instrument as the situation in which the first minimum of one interference pattern corresponds to the maximum of the other. This definition yields the following condition:

$$
\frac{\Delta N}{c}>\frac{\lambda^{2}}{\Delta \lambda c l_{2}}
$$

resulting in a minimum measurable polarization mode dispersion of $0.4 \mathrm{ps} / \mathrm{m}$ at $1300 \mathrm{~nm}$ and of $0.5 \mathrm{ps} / \mathrm{m}$ at $1550 \mathrm{~nm}$ using the above described setup $(\Delta \lambda=9.5 \mathrm{~nm}$, $\left.l_{2}=1.85 \mathrm{~m}\right)$. This technique is therefore clearly restricted to the measurement of fiber specially designed to be highly birefringent. If the condition (16) is fullfilled, the accuracy on polarization mode dispersion is estimated to be about $0.02 \mathrm{ps} / \mathrm{m}$. 


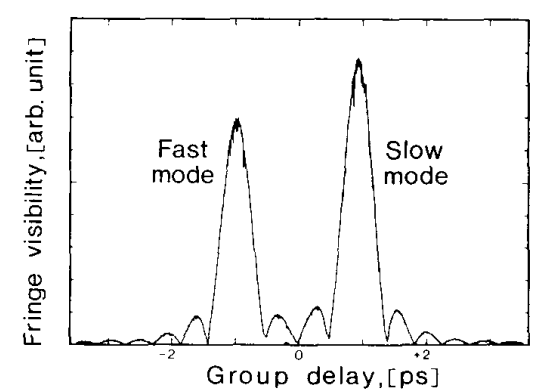

Fig. 2. Direct measurement of the interference visibility at $\lambda=1.3 \mu \mathrm{m}$ as a function of the delay between the interferometer arms when a highbirefringence fiber is in one arm, showing a distinct interference pattern for each polarization mode.

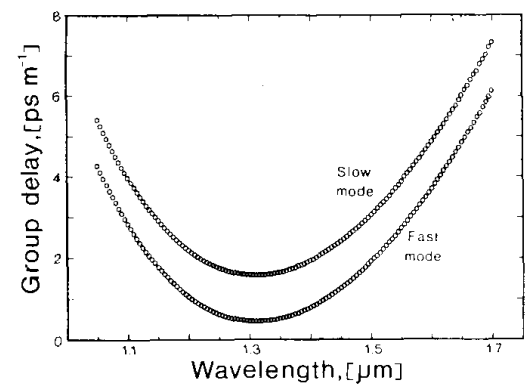

Fig. 3. Group delay spectrum of each polarization mode of a high-birefringence fiber.

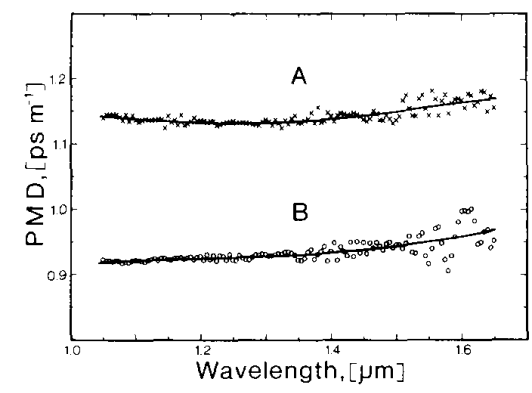

Fig. 4. Polarization mode dispersion (PMD) of two different high-birefringence fibers as a function of wavelength.

The birefringence $\Delta n$, however, can be evaluated in no way using such a technique. Nevertheless Fig. 4 clearly shows that the wavelength dependence of $\Delta N$ is very low. Actually the wavelength-independent stress-induced birefringence is much greater than the wavelength-dependent geometrically induced birefringence [8]. This observation is confirmed by other measurements on different fibers, even showing a strong core ellipticity [9]. Consequently the measurement of polarization mode dispersion, and thus $\Delta N$, yields an excellent approximation to the birefringence.

In order to measure fiber of lower birefringence, that is, when a strong overlapping of the interference patterns occurs, it remains possible to fit expression (14) on the measured visibility using a nonlinear least-squares method
[10]. But this technique requires a rather long computation time and may give unstable results. A better way is either to increase the source spectral width $\Delta \lambda$ resulting in a better separation of the interference patterns, or, if not sufficient, to strongly decrease the spectral width and to use the technique for low-birefringence fibers measurement described in the next section.

\section{B. Low-Birefringence Fibers}

A low birefringence in the test arm of the interferometer gives rise to a complete overlapping of the interference patterns of both the polarization modes, so that they are in no way distinguishable. In other words, owing to the slow variation of the contrast function $\left|g_{D}\right|$ in expression (14), the following approximation holds:

$$
\begin{aligned}
& \left|g_{D}\left[\beta_{1}^{\prime} l_{1}-\left(\beta_{2}^{\prime} l_{2}+\frac{\Delta N}{2 c}\right)\right]\right| \\
& \simeq\left|g_{D}\left[\beta_{1}^{\prime} l_{1}-\left(\beta_{2}^{\prime} l_{2}-\frac{\Delta N}{2 c}\right)\right]\right| \simeq\left|g_{D}\left[\beta_{1}^{\prime} l_{1}-\beta_{2}^{\prime} l_{2}\right]\right| .
\end{aligned}
$$

However, an equivalent approximation cannot be established for the cosine terms in (14) owing to their rapid variations. Using (17), expression (14) can be rewritten in the following form

$$
\begin{aligned}
I= & \frac{1}{2} \epsilon_{0} c\left|E_{0}\right|^{2}\left\{1+2\left|b_{1}\right|\left|b_{2}\right|\left|g_{D}\left[\beta_{1}^{\prime} l_{1}-\beta_{2}^{\prime} l_{2}\right]\right|\right. \\
& \left.\cdot \cos \left(\omega_{0} \frac{\Delta n}{2 c} l_{2}+\phi_{0}\right) \cdot \cos \left(\beta_{01} l_{1}-\beta_{02} l_{2}+\phi\right)\right\} .
\end{aligned}
$$

As intuitively anticipated, this expression shows that one interference pattern actually results from the interferometric reconstruction when the birefringence is low enough. But the birefringence effect gives rise to a periodic variation of the interference contrast when the optical frequency $\omega_{0}$ is scanned. Fig. 5 illustrates this feature showing actual interference visibility measurements of the same fiber at different wavelengths. The periodic spectral variation of the contrast is clearly observed when the maximum visibility is measured over a wide spectral range, as shown in Fig. 6. The period being directly related to the birefringence, it can be determined by performing a Fourier transform of the contrast as a function of the optical frequency. The result of such a transform is plotted in Fig. 7. The delay per unit length $\Delta n / c$ between the polarization modes, and thus the birefringence, is simply given by the abscissa of the sharp peak. The smaller peak is the first overtone and is just a computation artifact. A $23 \mathrm{ps} / \mathrm{km}$ delay between the polarization modes is obtained on this standard single-mode fiber and may result in a polarization mode dispersion exceeding the chromatic dispersion effect in normal conditions.

Contrary to the former method, the whole set of measurements over the spectrum is globally used to find the 


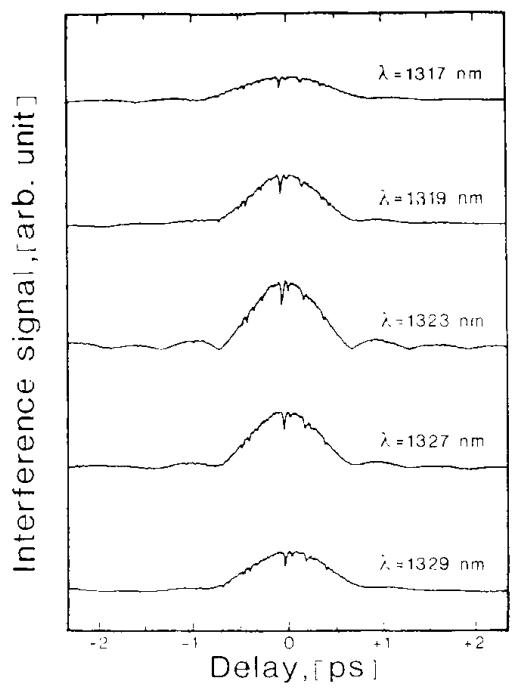

Fig. 5. Direct measurement of the interference visibility at different wavelengths when a low-birefringence fiber is in one arm, showing the contrast variations when the wavelength is scanned.

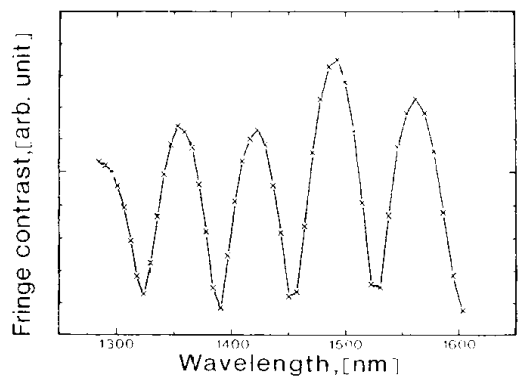

Fig. 6. Contrast spectrum showing periodic variations obtained using a low-birefringence fiber in the test arm.

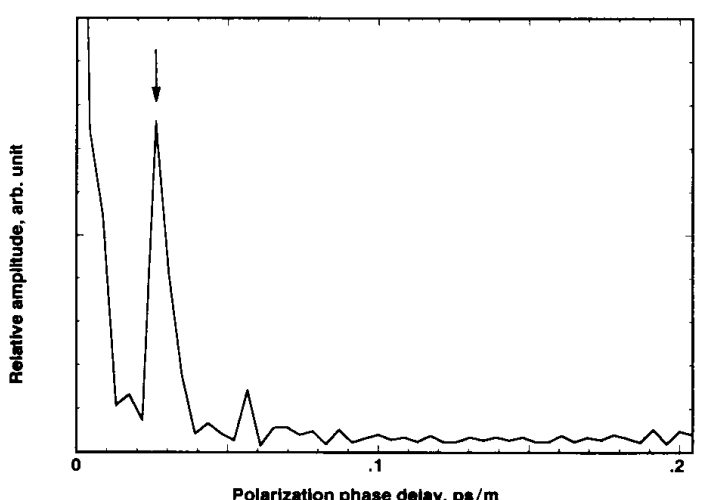

Fig. 7. Fourier transform of the contrast spectrum shown in Fig. 6. The phase delay between the polarization modes is given by the peak abscissa.

birefringence value, which must be assumed to be constant over the investigated spectral range. This assumption also implies $\Delta N=\Delta n$ and thus the polarization mode dispersion and the birefringence cannot be distinguished using this technique. This drawback is actually not a limitation, because the birefringence is mostly stress induced when accidental and thus wavelength independent, as previously mentioned and observed.

The resolution of this method is limited by the spread of the spectral range, because a full contrast oscillation must be measured to determine the period. Hence

$$
\Delta n_{\min }=\frac{\lambda_{\max } \lambda_{\min }}{l_{2}\left(\lambda_{\max }-\lambda_{\min }\right)}
$$

where $\lambda_{\min }, \lambda_{\max }$ are the lower and upper bounds of the investigated spectrum, respectively. The wide spectral range of the setup $\left(\lambda_{\min }=1100 \mathrm{~mm}, \lambda_{\max }=1700 \mathrm{~nm}\right)$ results in a $0.003 \mathrm{ps} / \mathrm{m}$ resolution on the delay between the polarization modes. This is more than two orders of magnitude better than the former technique.

This resolution makes the method perfectly suitable for systematic fiber controls before the installation of an optical link. However, it corresponds to a beat length at 1300 $\mathrm{nm}$ shorter than $1.5 \mathrm{~m}$ and is therefore not sufficient to detect polarization effects and phase retardation in fibers such as those involved in sensors applications.

\section{Conclusion}

These two methods for birefringence measurements are particularly suitable for a systematic control of either highor low-birefringence fibers to be used as transmission links. This is achieved using an unpolarized light and no polarizing device, so that the birefringence eigenaxes do not have to be matched to the polarization state of the incoming light. Linear and circular, or even elliptical, birefringences give the same experimental response and can therefore be equally measured with no setup modifications. Furthermore, a chromatic dispersion measurement can be simultaneously performed using the same experimental configuration and even the same interference visibility measurements. These advantages together with the wide spectral coverage of the setup intend this interferometric method to be a very universal measuring tool for single-mode fibers.

\section{REFERENCES}

[1] I. P. Kaminow, "Polarization in optical fibers," IEEE J. Quantum Electron., vol. QE-17, pp. 15-22, 1981

[2] S. C. Rashleigh and R. Ulrich, "Polarization mode dispersion in single-mode fibers," Opt. Lett., vol. 3, pp. 60-62, 1978.

[3] T. Okoshi, S. Ryu, and K. Emura, "Measurement of polarization parameters of single-mode optical fiber," J. Opt. Commun., vol. 2 , pp. 134-141, 1981.

[4] M. Monerie, P. Lamonir, and L. Veunhomme, "Polarization mode dispersion measurements in long single-mode fibers, " Electron. Lett., vol. 16, pp. 907-908, 1980

[5] K. Mochizuki, Y. Namihira, and H. Wakabayashi, "Polarization mode dispersion measurements in long single-mode fibers," Elec tron. Lett., vol. 17, pp. 153-154, 1981.

[6] N. Shibata, M. Tateda, and S. Seikai, "Polarization mode dispersion measurement in elliptical core single-mode fibers by a spatial technique," IEEE J. Quantum Electron., vol. QE-18, pp. 53-58, 1982.

[7] L. Thévenaz, J. P. Pellaux, and J. P. von der Weid, "All-fiber interferometer for chromatic dispersion measurements," J. Lightwave Technol., vol. 6, pp. 1-7, 1988 
[8] S. C. Rashleigh, "Origins and control of polarization effects in single-mode fibers," J. Lightwave Technol., vol. LT-1, pp. 312-331, 1983.

[9] J. P. von der Weid, L. Thévenaz, and J. P. Pellaux, "Interferometric measurements of chromatic and polarization mode dispersion in highly birefringent single-mode fibers," Electron. Lett., vol. 23, pp. 151$152,1987$.

[10] L. Thévenaz, V. de Coulon, and J. P. von der Weid, "Polarization mode interferometry in birefringent single-mode fibers," Opt. Lett., vol. 12 , pp. 619-621, 1987.

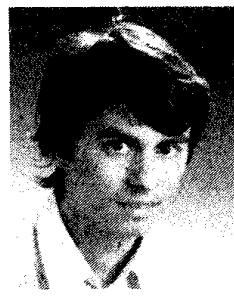

Luc Thévenaz received the B.S. degree in astrophysics from the Observatory of Geneva, Geneva, Switzerland, in 1982, and the Ph.D. degree in physics from the University of Geneva in 1988.

In 1983 he joined the Group of Applied Physics at the University of Geneva, Geneva, Switzerland, where he was engaged in research on singlemode fiber measurement techniques and where he developed new methods for chromatic dispersion and birefringence measurements. Early in 1988 , he joined the Laboratory of Metrology of the Swiss

Federal Institute of Technology in Lausanne, Switzerland, where he is Lecturer and presently occupies a postdoctoral position. His current interests are in characterization of optical guided-wave devices and in their applications.

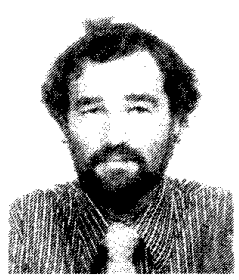

Jean-Paul Pellaux received the Ph.D. degree in physics from the University of Neuchâtel, Switzerland, in 1978 .

He joined the Microtechnique Institute of the University of Neuchâtel working on holographic coupling of optical fibers. Later he was head of the Research and Development Staff in Cabloptic Fiber Manufacture. In 1982 he joined the Applied Physics Group. University of Geneva, Geneva, Switzerland. He is now Manager of the Development Group in Alphatronix.

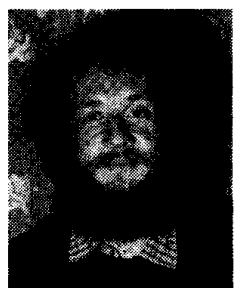

Nicolas Gisin was born in Geneva, Switzerland, in 1952 . He received the Ph.D. degree in physics from the University of Geneva in 1982 for his dissertation on quantum and statistical physics.

In 1983 he joined the University of Rochester. In 1985 he joined the Alphatronix company. Since 1988 he has been with the Group of Applied Physics at the University of Geneva. His research work ranges from quantum physics to fiber optics and digital image processing.

Dr. Gisin received the Dina Surdin Award in 1983 from the Fondation Louis de Broglie.

Jean-Pierre von der Weid received the Ph.D. degree in physics from Pontificia Universidade Catholica (PUC), Rio De Janeiro, Brazil, in 1975.

He joined the Physics Institute, University of Neuchâtel, Switzerland, working on solid-state physics. He then returned as Professor to the Physics Department of PUC University, Rio de Janeiro, Brazil. He spent one year in 1986 with the Applied Physics Group, University of Geneva, Switzerland, where he investigated chromatic dispersion and birefringence measurement techniques. 